\title{
Applying Linear Elastic Glucose Behavior Theory and AI Auto-Correction to Predict A1C Variances over the Ninth Period Using GH-Method: Math-Physical Medicine (No. 354)
}

\author{
Gerald C. Hsu* \\ eclaire MD Foundation, 10/28-29/2020, USA \\ ${ }^{\star}$ Corresponding author: Gerald C. Hsu, eclaire MD Foundation, 10/28-29/2020, USA
}

Received: November 05, 2020; Accepted: November 07, 2020; Published: November 09, 2020

\begin{abstract}
In this case study, the author analyzed, predicted, and interpreted a type 2 diabetes (T2D) patient's hemoglobin A1C variance or A1C value based on nine time periods, $\sim 5$ months each, utilizing the GH-Method: math-physical medicine (MPM) approach. He utilized the same method and calculation formulas since 1/1/2014. This particular article emphasizes the ninth period (Period I) ranging from 5/20/2019 to 10/21/2020.

Unlike the previous eight periods, in the ninth period, he used the continuous glucose monitor (CGM) sensor collected glucose data, which he implemented with the recently developed linear elastic glucose behavior theory (Reference 7, No. 352) to calculate his postprandial plasma glucose (PPG) difference.

Utilizing an auto-learning and auto-correction technique of artificial intelligence (AI), his software system would learn from lab-tested A1C values and then make necessary corrections. Therefore, in Period I, he utilized a higher conversion factor of 17.13 from glucose to HbA1C instead of 12.5 in the previous seven periods (Period A throughout Period G). He wrote two articles for the eighth period (Period H) using 12.5 (No. 329) and 17.13 (No. 329B), respectively. The difference on the final $\mathrm{HbA} 1 \mathrm{C}$ results is a mere $0.01 \%$ which increased his predicted $\mathrm{HbA} 1 \mathrm{C}$ from $6.31 \%$ to $6.39 \%$, while the lab-tested $\mathrm{HbA1C}$ was $6.4 \%$.
\end{abstract}

There are 10 hemoglobin $\mathrm{A} 1 \mathrm{C}$ lab-tested results, with his 10 predicted $\mathrm{HbA1C}$ values using his developed methods:

- $6.7 \%$ on $4 / 9 / 2017$ (predict $6.7 \%$ )

- $6.1 \%$ on $9 / 12 / 2017$ (predict $6.1 \%$ )

- $6.9 \%$ on $1 / 26 / 2018$ (predict $6.9 \%$ )

- $6.5 \%$ on $6 / 29 / 2018$ (predict $6.5 \%$ )

- $6.6 \%$ on $10 / 22 / 2018$ (predict $6.6 \%$ )

- $6.8 \%$ on $4 / 4 / 2019$ (predict $6.8 \%$ )

- $6.6 \%$ on $9 / 25 / 2019$ (predict $6.6 \%$ )

- $6.6 \%$ on $12 / 20 / 2019$ (predict $6.6 \%$ )

- $6.4 \%$ on $5 / 20 / 2019$ (predict $6.4 \%$ )

- $6.2 \%$ on $10 / 21 / 2020$ (predict $6.2 \%$ ).

It should be pointed out that all of his 10 predicted $\mathrm{HbA1C}$ values match 100\% with his lab-tested $\mathrm{HbA1C}$ values.

The author focused on nine periods over 1,299 days. It contains 3,897 meal data, including key contribution factors such as carbs/sugar intake, post-meal exercise, weather, and more. This study demonstrated a high degree of accuracy for the calculation and prediction of the patient's forthcoming HbA1C value by using the GH-Method: math-physical medicine approach. In this article, the author also utilized CGM sensor glucose data with AI-tuned conversion factor between glucose and HbA1C. Furthermore, he also implemented his developed linear elastic glucose behavior theory (Reference 7) for his PPG calculation in order to obtain a better estimation for the final HbA1C value.

Once the healthcare professionals and T2D patients understand the $\mathrm{HbA} 1 \mathrm{C}$ mathematical prediction method, then the overall diabetes condition for the patient can be easier to control. The purpose for this research paper is to help people with T2D by preventing further damage to their internal organs caused by elevated $\mathrm{A} 1 \mathrm{C}$ values, before taking the laboratory test. 
If healthcare professionals and diabetes patients have an interest to delve deeper regarding the formation of tested glucose and mathematical predicted A1C, they should focus on the influential factors and their respective weighted contribution percentages described in the author's previous papers.

Here is the summary:

1. The most important month which contributes to the $\mathrm{A} 1 \mathrm{C}$ is the month prior to the lab test.

2. PPG contribute $>2 / 3$ of $\mathrm{HbA} 1 \mathrm{C}$.

3. Body weight controls $\sim 77 \%$ or more of the fasting plasma glucose (FPG) which contributes $<1 / 3$ of HbA1C; therefore, it is important to keep BMI below 25.

4. Carbs/sugar amount contributes $\sim 39 \%$ to PPG. For T2D patients, it is safe to keep carbs/sugar intake amount below 15 grams per meal.

5. Post-meal walking steps contributes $\sim 41 \%$ to PPG. It is recommended to maintain post-meal walking exercise around 4,000 steps after each meal.

6. A combined effort of diet and exercise controls $\sim 80 \%$ to PPG formation.

\section{Introduction}

In this case study, the author analyzed, predicted, and interpreted a type 2 diabetes (T2D) patient's hemoglobin A1C variance or A1C value based on nine time periods, $\sim 5$ months each, utilizing the GHMethod: math-physical medicine (MPM) approach. He utilized the same method and calculation formulas since $1 / 1 / 2014$. This particular article emphasizes the ninth period (Period I) ranging from 5/20/2019 to $10 / 21 / 2020$.

Unlike the previous eight periods, in the ninth period, he used the continuous glucose monitor (CGM) sensor collected glucose data, which he implemented with the recently developed linear elastic glucose behavior theory (Reference 7, No. 352) to calculate his postprandial plasma glucose (PPG) difference.

Utilizing an auto-learning and auto-correction technique of artificial intelligence (AI), his software system would learn from labtested A1C values and then make necessary corrections. Therefore, in Period I, he utilized a higher conversion factor of 17.13 from glucose to $\mathrm{HbA1C}$ instead of 12.5 in the previous seven periods (Period A throughout Period G). He wrote two articles for the eighth period
(Period H) using 12.5 (No. 329) and 17.13 (No. 329B), respectively. The difference on the final $\mathrm{HbA1C}$ results is a mere $0.01 \%$ which increased his predicted $\mathrm{HbA} 1 \mathrm{C}$ from $6.31 \%$ to $6.39 \%$, while the labtested $\mathrm{HbA1C}$ was $6.4 \%$ [1-7].

\section{Method}

As shown in Figure 1, there are 10 hemoglobin A1C lab-checkup results, with his 10 predicted $\mathrm{HbA1C}$ values using his developed methods:

- $6.7 \%$ on $4 / 9 / 2017$ (predict $6.7 \%$ )

- $6.1 \%$ on $9 / 12 / 2017$ (predict $6.1 \%$ )

- $6.9 \%$ on $1 / 26 / 2018$ (predict $6.9 \%$ )

- $6.5 \%$ on $6 / 29 / 2018$ (predict $6.5 \%$ )

- $6.6 \%$ on $10 / 22 / 2018$ (predict $6.6 \%$ )

- $6.8 \%$ on $4 / 4 / 2019$ (predict $6.8 \%$ )

- $6.6 \%$ on $9 / 25 / 2019$ (predict $6.6 \%$ )

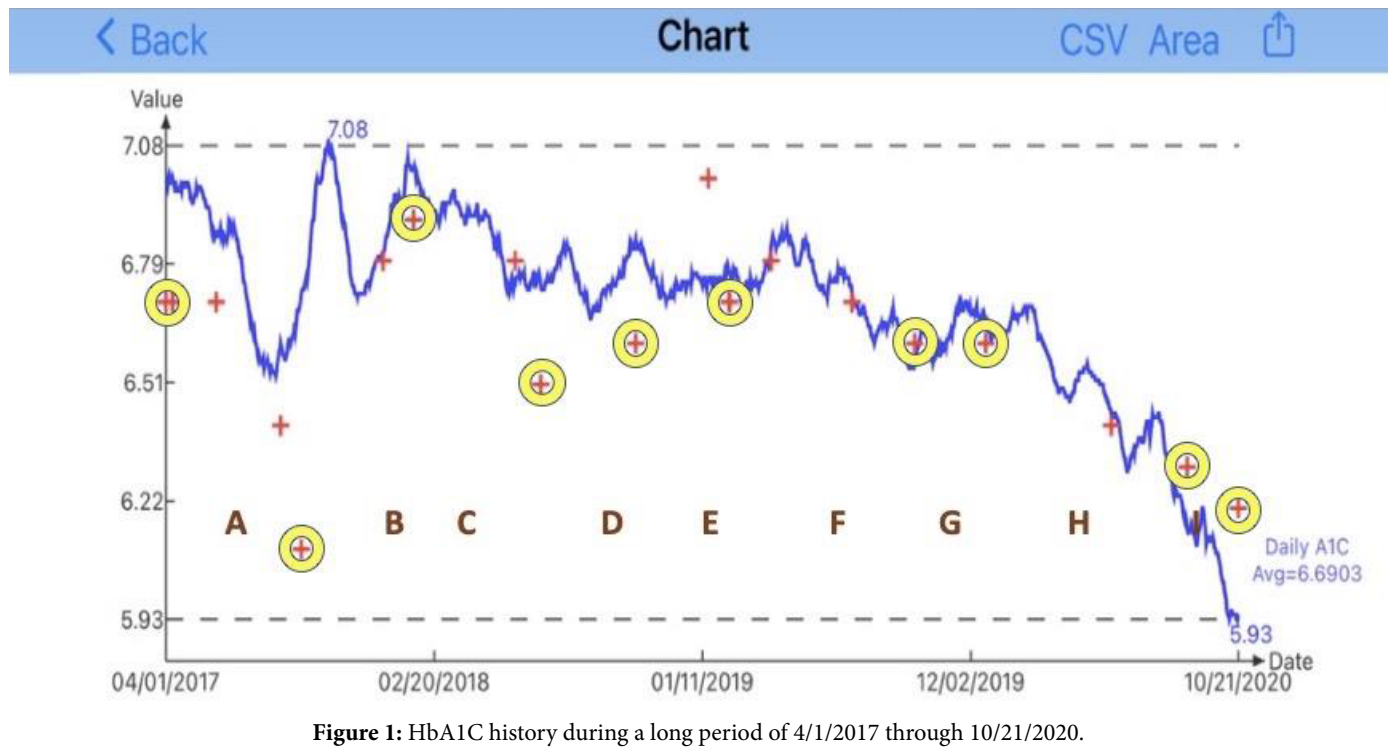


- $6.6 \%$ on $12 / 20 / 2019$ (predict $6.6 \%$ )

- $6.4 \%$ on $5 / 20 / 2019$ (predict $6.4 \%$ )

- $6.2 \%$ on $10 / 21 / 2020$ (predict $6.2 \%$ ).

It should be pointed out that all of his 10 predicted $\mathrm{HbA1C}$ values match 100\% with his lab-tested HbA1C values.

The author selected nine periods of almost equal length with $\sim 5$ months each, then observed their measured A1C changes (variances) against the previous period as follows:

- Period A (4/1/2017 - 8/31/2017): -0.6\%

- Period B (9/1/2017 - 1/31/2018): +0.8\%

- Period C (2/1/2018 - 6/30/2018): $-0.4 \%$

- Period D (6/29/2018 - 10/22/2018): $+0.1 \%$

- Period E (10/22/2018 - 4/4/2019): +0.2\%

- Period F (4/4/2019 - 9/25/2019): -0.2\%

- Period G (9/25/2019 - 12/20/2019): +0.0\%

- Period H (12/20/2019 - 5/20/2020): -0.2\%

- Period I (5/20/2020 - 10/21/2020): -0.2\%.

He applied his developed GH-Method: math-physical medicine approach along with the following seven contribution factors of HbA1C:

1. A1C variances contributed by FPG between $15 \%$ to $35 \%$, where he used $25 \%$ in his calculation for this article.

2. FPG variance due to weight change with $\sim 77 \%$ contribution.

3. Colder weather impact on FPG with a decrease of each Fahrenheit degree caused $0.3 \mathrm{mg} / \mathrm{dL}$ decrease of FPG.

4. A1C variances contributed by PPG between $65 \%$ to $85 \%$, where he used $75 \%$ in his calculation for this article.

5. PPG variance due to carbs/sugar intake with $\sim 39 \%$ weighted contribution on PPG.

6. PPG variance due to post-meal walking with $\sim 41 \%$ weighted contribution on PPG.

7. Warm weather impact on PPG with an increase of each Fahrenheit degree caused $0.9 \mathrm{mg} / \mathrm{dL}$ increase of PPG.

It should be noted that his developed mathematical HbA1C prediction model is based on different weighted ratio for the previous 4-month glucose data, instead of the standard concept of the threemonth average glucose for $A 1 C$ with equal weighting factors. He chose 120 days for his HbA1C calculation which is based on the fact that the average human red blood cells (RBC), after differentiating from erythroblasts in the bone marrow, are released into the blood and survive in circulation for approximately 115 days.
In 2019, he developed the following simple linear equation for PPG prediction:

Predicted $P P G=($ measured $F P G * 0.97)+($ carbs/sugar intake amount ${ }^{*}$ GH-modulus) - (post-meal walking K-steps $\left.{ }^{*} 5\right)$.

Where $97 \%$ of FPG can be served as the baseline PPG.

In 2020, after re-arranging the above equation into the following two new terms with a newly defined coefficient, the "GH-modulus":

$\mathrm{X}=$ carbs/sugar amount

$\mathrm{Y}=$ measured PPG $-\left(\mathrm{FPG}^{\star}{ }^{\star 0.97}\right)+($ post-meal walking $\mathrm{k}$-steps *5)

where $\mathrm{X}$ functions as the input, stress, or stimulator on the liver, and $\mathrm{Y}$ functions as the output, strain, or consequence from the liver. Both $\mathrm{X}$ input and $\mathrm{Y}$ output are related to each other through the coefficient of GH-modulus, as shown below:

GH-modulus $=(Y$ output $) /(X$ input $)$

in endocrinology of biomedical science

and

Young's modulus $(E)=$ stress/strain

in linear elasticity of engineering strength of materials.

The author has applied this linear elastic glucose theory in his PPG calculation for estimating his $\mathrm{HbA1C}$ value in the ninth period.

Please note that other than his acquired biomedical knowledge and his findings from previous research work, there are no other complicated or sophisticated mathematical tools being used in this analysis.

\section{Results}

Based on the author's numerous publications of $\mathrm{HbA1C}$ contributions by FPG and PPG, along with the prediction models of these two glucoses and $\mathrm{HbAlC}$, a summarized chart of these $\mathrm{HbA1C}$ values over nine periods from 4/1/2017 to $10 / 21 / 2020$ are observed in Figure 1.

This long period of $\sim 3.5$ years, $\sim 43$ months, 1,299 days are divided into nine periods of almost equal length of 4.6 months. Although his calculated daily A1C data and curves are fluctuating, the 10 labcheckup dates with two sets of A1C values (predicted A1C and labtested $A 1 C$ ) are identical to each other.

Figure 2 shows the step-by-step background data table of Period I. During this time, his primary input data for the "linear elastic glucose equation" are:

$$
\begin{aligned}
& \text { Average } F P G=100.84 \mathrm{mg} / \mathrm{dL} \\
& \text { Average carbs }=12.24 \text { grams } \\
& \text { GH-modulus }=3.9(\text { Reference } 7) \\
& \text { Averaged walking }=4.122 \mathrm{k} \text {-steps }
\end{aligned}
$$




\begin{tabular}{|c|c|c|c|c|c|c|c|c|}
\hline Peried & Date: From & Date: To & Wedght Change & FPG: From avg & FPG: To end & \begin{tabular}{|l|} 
FPG Change \\
\end{tabular} & FPG $(25 \%)$ & $\mathrm{AIC}$ (FPG) $a 17.13$ \\
\hline G & 9/25/19 & $12 / 20 / 19$ & 1 & 111 & 119 & 8 & 2.0 & 0.16 \\
\hline H & $12 / 20 / 19$ & $5 / 20 / 20$ & -2 & 120 & 110 & -10 & -2.5 & -0.15 \\
\hline I & $520 / 20$ & $10 / 21 / 20$ & -6 & 101 & 97 & -4 & -1.0 & -0.06 \\
\hline Period & Date: From & Date: To & Carbs \& Walking & PPG: From start & PPG: To pred avg & PPG Change & PPG (75\%) & $\mathrm{AIC}(\mathrm{PPG})=17.13$ \\
\hline G & $9 / 25 / 19$ & $12 / 20 / 19$ & $+4 / 300$ & 110 & 109 & -1 & -0.8 & -0.06 \\
\hline H & $12 / 20 / 19$ & $5 / 20 / 20$ & $-3.5 /-100$ & 111 & 110 & -1 & -1.1 & -0.06 \\
\hline I & $5 / 20 / 20$ & $10 / 21 / 20$ & & 128 & 125 & -3 & -2.4 & -0.14 \\
\hline Period & Lab Dates & Lab Renults & Lab (AIC Change) & $\mathrm{AIC}(\mathrm{F})$ & $\overline{A I C(P P G)}$ & AIC Variance & ectalin:MD $\mathrm{AIC} \%$ & \\
\hline G & $9 / 25 / 19$ & $6.6 \%$ & $-0.2 \%$ & 0.16 & -0.06 & 0.10 & $6.7 \%$ & \\
\hline $\mathrm{H}$ & $12 / 10 / 19$ & $6.6 \%$ & $0.0 \%$ & -0.15 & -0.06 & -0.21 & $6.39 \%$ & \\
\hline I & $5 / 20 / 20$ & $6.4 \%$ & $-0.2 \%$ & -0.06 & -0.14 & -0.20 & $6.2 \%$ & \\
\hline $\mathrm{J}$ & $10 / 21 / 20$ & $6.2 \%$ & & & & & & \\
\hline & GH-modulus & Avg FPG & Avg Carbs & Avg Walking & Pred. Avg PPG & & & \\
\hline & 3.9 & 100.84 & 12.24 & 4.122 & 124.8 & & & \\
\hline
\end{tabular}

\begin{tabular}{|c|c|c|c|c|c|c|c|c|c|c|}
\hline Male Sensor & $1 / 18-2 / 18$ & $2 / 18-3 / 18$ & $3 / 18-4 / 18$ & $4 / 18-5 / 18$ & $5 / 18-6 / 18$ & $6 / 18-7 / 18$ & $7 / 18-8 / 18$ & $8 / 18-9 / 18$ & $9 / 18-10 / 18$ & Average \\
\hline PPG (peak) & 134.90 & 135.13 & 140.83 & 133.37 & 128.06 & 134.16 & 120.46 & $\mathbf{1 2 5 . 4 3}$ & 119.47 & 130.20 \\
\hline Male Measured PPG (Average) & 126 & 122 & 136 & 125 & 117 & 128 & 115 & 119 & 112 & 122 \\
\hline FPG (Average) & 115.11 & 97.31 & 113.58 & 104.81 & 96.11 & 100.55 & 100.23 & 99.18 & 9108 & 102.00 \\
\hline Carbs/Sugar (grams) & 10.54 & 13.15 & 12.73 & 16.18 & 12.55 & 9.79 & 10.67 & 12.93 & 1181 & 12.26 \\
\hline Walking (K-steps) & 4.519 & 4.763 & 4.528 & 4.268 & 3.895 & 4.039 & 4.009 & 4.148 & 4.252 & 4.269 \\
\hline Male X: (Carbs"M2) & 37.07 & 51.29 & 48.02 & 44.94 & 43.53 & 50.85 & 38.14 & 43.80 & 45.04 & 44.74 \\
\hline Male Y: (Meas. PPG) $-(0.97 \mathrm{FPG})+($ Steps $* 5)$ & 37.07 & 51.29 & 48.02 & 44.94 & 43.53 & 50.85 & 38.14 & 43.80 & 45.04 & 44.74 \\
\hline Male Annual M2 (Variable) & 3.5 & 3.9 & 3.8 & 2.8 & 3.5 & 5.2 & 3.6 & 3.4 & & \\
\hline
\end{tabular}

Figure 2: HbA1C step-by-step calculation table during 9 periods (4/1/2017 - 10/21/2020).

Predicted $P P G=($ measured $F P G * 0.97)+($ carbs/sugar intake amount ${ }^{*}$ GH-modulus) - (post-meal walking K-steps $\left.{ }^{*} 5\right)$

Therefore,

Predicted average PPG in Period I

$=\left(100.84^{\star} 0.97\right)+\left(12.24^{\star} 3.9\right)-\left(4.122^{\star} 5\right)$

$=125 \mathrm{mg} / \mathrm{dL}$

This value has been used in the calculation of obtaining the $6.2 \%$ $\mathrm{HbA1C}$ value in the ninth period.

Figure 3 depicts his weight, carbs/sugar intake, post-meal walking, sensor FPG, and sensor PPG in Period I from 5/20/2020 to 10/21/2020.

\section{Conclusion}

The author focused on nine periods over 1,299 days. It contains 3,897 meal data, including key contribution factors such as carbs/ sugar intake, post-meal exercise, weather, and more. This study demonstrated a high degree of accuracy for the calculation and prediction of the patient's forthcoming $\mathrm{HbA1C}$ value by using the GH-Method: math-physical medicine approach. In this article, the author also utilized CGM sensor glucose data with AI-tuned conversion factor between glucose and HbA1C. Furthermore, he also implemented his developed linear elastic glucose behavior theory (Reference 7) for his PPG calculation in order to obtain a better estimation for the final HbA1C value.

Once the healthcare professionals and T2D patients understand the $\mathrm{HbAlC}$ mathematical prediction method, then the overall diabetes condition for the patient can be easier to control. The purpose for this research paper is to help people with T2D by preventing further damage to their internal organs caused by elevated A1C values, before taking the laboratory test.
If healthcare professionals and diabetes patients have an interest to delve deeper regarding the formation of tested glucose and mathematical predicted $\mathrm{A} 1 \mathrm{C}$, they should focus on the influential factors and their respective weighted contribution percentages described in the author's previous papers.

Here is the summary:

1. The most important month which contributes to the A1C is the month prior to the lab test.

2. PPG contribute $>2 / 3$ of $\mathrm{HbA} 1 \mathrm{C}$.

3. Body weight controls $\sim 77 \%$ or more of the fasting plasma glucose (FPG) which contributes $<1 / 3$ of HbA1C; therefore, it is important to keep BMI below 25 .

4. Carbs/sugar amount contributes $\sim 39 \%$ to PPG. For T2D patients, it is safe to keep carbs/sugar intake amount below 15 grams per meal.

5. Post-meal walking steps contributes $\sim 41 \%$ to PPG. It is recommended to maintain post-meal walking exercise around 4,000 steps after each meal.

6. A combined effort of diet and exercise controls $\sim 80 \%$ to PPG formation.

\section{References}

1. Hsu, Gerald C. eclaireMD Foundation, USA. "Biomedical research methodology based on GH-Method: math-physical medicine (No. 310).”

2. Hsu, Gerald C. eclaireMD Foundation, USA. "A Case Study on the Prediction of A1C Variances over Seven Periods with guidelines Using GH-Method: math-physical medicine (No. 262)."

3. Hsu, Gerald C. eclaireMD Foundation, USA. "A Case Study on the Investigation and Prediction of AlC Variances Over Six Periods Using GH-Method: math-physical medicine (No. 116)." 


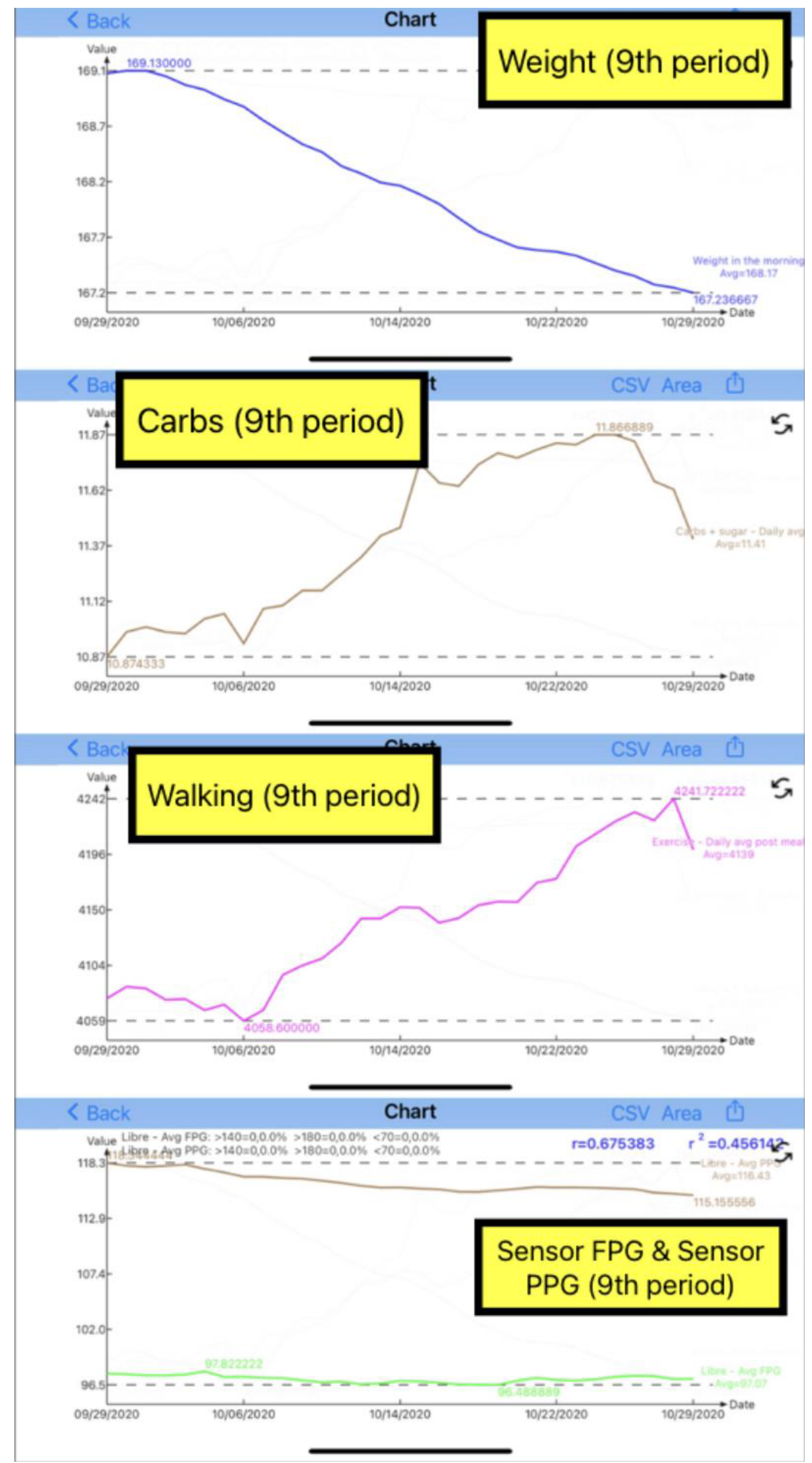

Figure 3: Weight, carbs/sugar intake, post-meal walking, sensor FPG, and sensor PPG during the ninth period (period I from 5/20/2020 to 10/21/2020). 
Gerald C. Hsu (2020) Applying Linear Elastic Glucose Behavior Theory and AI Auto-Correction to Predict A1C Variances over the Ninth Period Using GH-Method: Math-Physical Medicine (No. 354)

4. Hsu, Gerald C. eclaireMD Foundation, USA. "A Case Study of Investigation and Prediction of AlC Variances Over 5 Periods Using GH-Method: math-physical medicine (No. 65)."

5. Hsu, Gerald C. eclaireMD Foundation, USA. "Segmentation and pattern analyses for three meals of postprandial plasma glucose values and associated carbs/sugar amounts using GH-Method: math-physical medicine (No. 326)."
6. Hsu, Gerald C. eclaireMD Foundation, USA. "Using GH-Method: math-physical medicine to Conduct Segmentation Analysis to Investigate the Impact of both Weight and Weather Temperatures on Fasting Plasma Glucose (No. 68)."

7. Hsu, Gerald C. eclaireMD Foundation, USA. "Investigation of linear elastic glucose behavior with GH-modulus linking carbohydrates/sugar intake and incremental PPG via an analogy of Young's modulus from theory of elasticity and engineering strength of materials using GH-Method: math-physical medicine, Parts 1, 2, and 3 (No. 352)."

Gerald C. Hsu (2020) Applying Linear Elastic Glucose Behavior Theory and AI Auto-Correction to Predict A1C Variances over the Ninth Period Using GH-Method: Math-Physical Medicine (No. 354). Endocrinol Diabetes Metab J Volume 4(5): 1-6. 